\title{
Acute Eosinophilic Pneumonia Due to Vaping-Associated Lung Injury
}

\author{
Molly Wolf*, Jeremy Richards \\ Beth Israel Deaconess Medical Center, Boston, MA, USA
}

\begin{abstract}
A case is described of a 29-year-old female who presented with acute hypoxic respiratory failure due to acute eosinophilic pneumonia, associated with the use of electronic cigarettes to vape tetrahydrocannabinol (THC), together with the contemporary clinical understanding of the syndrome of electronic-cigarette associated lung injury (EVALI). Attention is drawn to acute eosinophilic pneumonia as a potential consequence of vaping-associated lung injury to understand the diagnostic evaluations and therapeutic interventions for acute eosinophilic pneumonia associated with vaping THC.
\end{abstract}

Keywords: acute hypoxic respiratory failure, acute eosinophilic pneumonia, tetrahydrocannabinol (THC), electroniccigarette associated lung injury, EVALI

Received: 18 February 2020 / Accepted: 16 October 2020

\section{INTRODUCTION}

Acute eosinophilic pneumonia (AEP) is a rare clinical disease that has been described in young patients between 20-40 years of age, and more commonly in men $[1,2]$. Patients usually present with less than four weeks of symptoms, most commonly including non-productive cough, dyspnea, and fever.

AEP has also been rarely reported as an adverse effect from vaping e-cigarettes $[3,4]$.

\section{CASE REPORT}

A previously healthy 29-year-old female was admitted to Beth Israel Hospital in Boston, MA USA. On admission, she reported that she had a current alcohol use disorder, drinking 5-10 drinks per day for the last ten years or so, and excessive tobacco use which she stopped six months before admission.

She started using non-flavoured product THC-containing e-cigarettes ("vape") four weeks before admission. The THC-containing e-cigarette product was purchased from a local licensed marijuana dispensary.

Otherwise, she had been well until one week prior to admission, when she developed profound fatigue. Four days before admission, she developed a sore throat; and one day before admission, she developed subjective fevers and shortness of breath.
On arrival at the hospital, she was afebrile with an oxygen saturation of $71 \%$ on a non-rebreather mask. She was in significant respiratory distress, and pulmonary examination revealed diffuse rhonchi bilaterally with bibasilar dullness. Her white blood cell count was $15.3 ; 88 \%$ neutrophils and 5.8\% eosinophils. A chest radiograph, taken on the day of admission, demonstrated extensive pulmonary opacities bilaterally (Figure 1). A chest CT scan, on the same day, demonstrated diffuse, bilateral broncho-centric disease including nodular ground-glass opacities coalescing to form consolidation at the bases (Figure 2).

She was immediately admitted to the hospital's ICU and intubated for refractory hypoxemia.

On Day 1 of hospitalization, sputum culture was negative for viral and bacterial pathogens, and a bronchoscopy was performed. The cell count from her bronchoalveolar lavage (BAL) revealed 36\% eosinophils, and the cytopathology demonstrated foamy macrophages (Figure 3). Based on these tests, she was diagnosed with acute eosinophilic pneumonia by the critical care physicians, as defined by criteria in Table $1[1,2,5,6]$.

Twenty-four hours after admission, she was started on high-dose steroids, $500 \mathrm{mg}$ per day of intravenous methylprednisone (Pfizer, New York, US), given intravenously.

By Day 2, chest radiographs showed a reduction of pulmonary infiltrates. There was a rapid improvement 
260 - The Journal of Critical Care Medicine 2020;6(4)

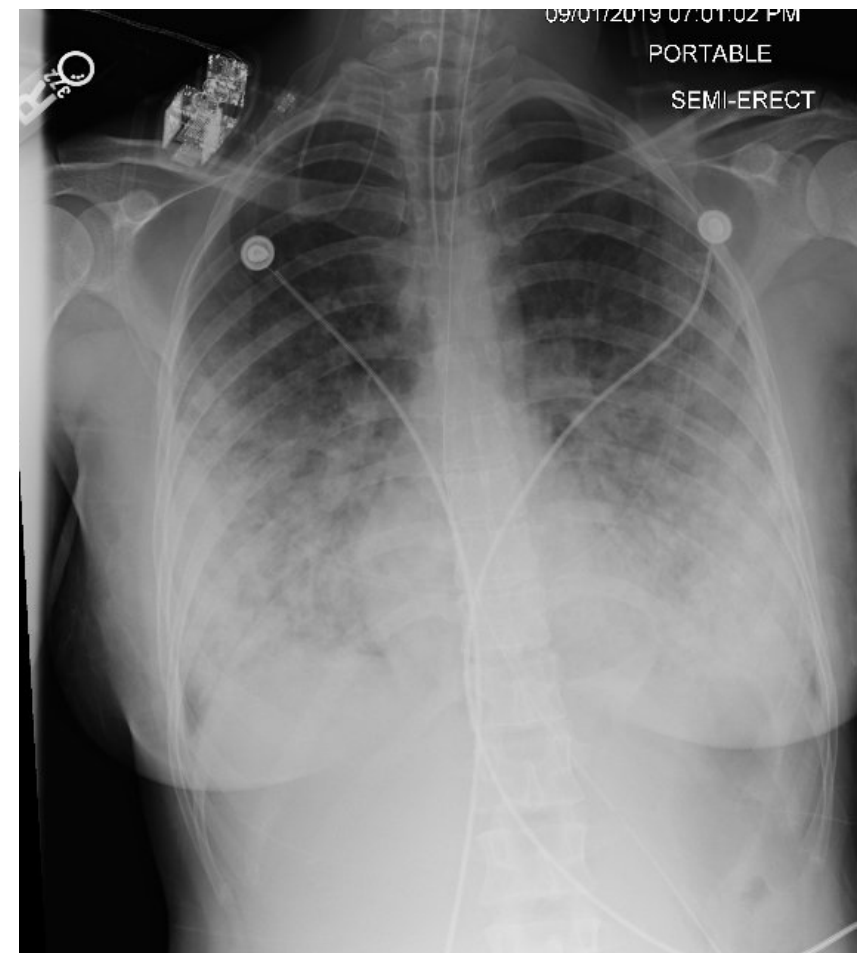

Fig. 1. Chest radiograph (CXR) demonstrating bilateral peripheral and central opacities with a basilar predominance

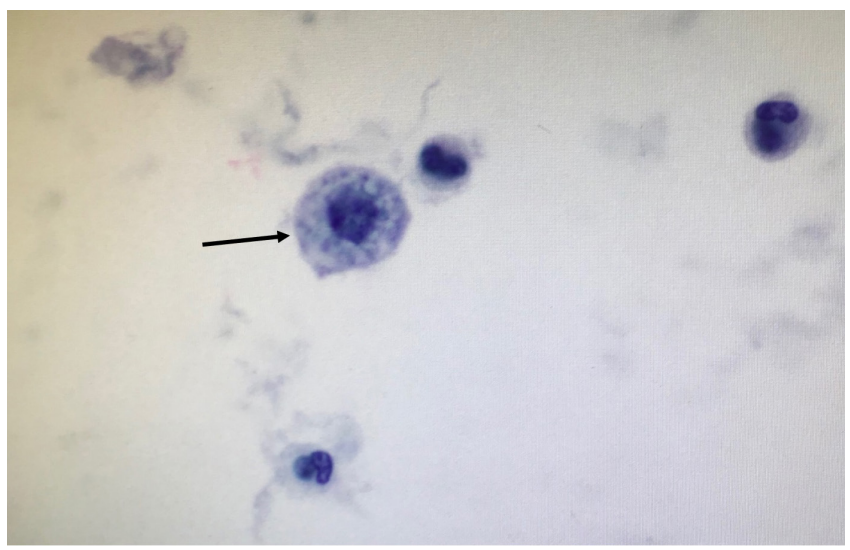

Fig. 3. Lung cytopathology, demonstrating rare foamy macrophages (black arrow).

in hypoxemia, allowing for extubation on Day 3. Her steroids were tapered to oral prednisone, $1 \mathrm{mg} / \mathrm{kg}$ per day (American Health Packaging, Columbus, US).
Available online at: www.jccm.ro

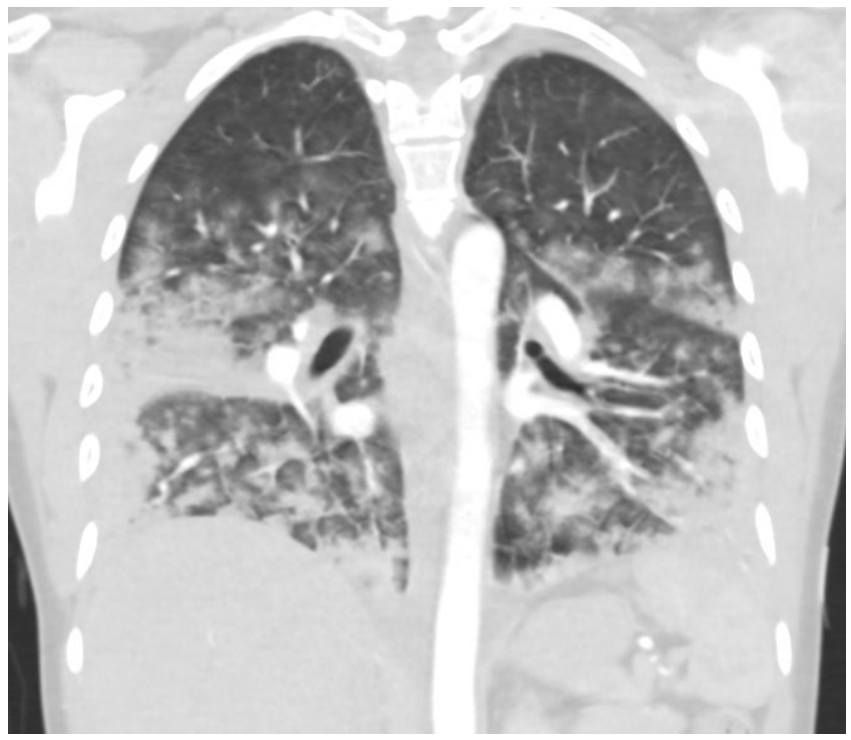

Fig. 2. Chest computerized tomography (CT) demonstrating diffuse, broncho-centric bilateral ground glass opacities with coalescence to consolidation, largely in the lung bases

She was discharged home on Day 7 with exertional oxygen supplementation and plans for a follow-up after two weeks in the hospital's outpatient pulmonary clinic.

\section{DISCUSSION}

The criteria for making a clinical diagnosis of AEP are listed in Table $1[1,2,5,6]$. This includes a BAL with a cell count demonstrating $>25 \%$ eosinophilia. These criteria were observed in the present case. They are more commonly described in men [2]. A thorough evaluation should include a review of medications, prior chest irradiation, and travel to areas with endemic parasites. Patients typically have a normal peripheral blood eosinophilia on presentation, although this can rise later.

The differential diagnosis for AEP is delineated in Table $2[1,7,8,9,10]$.

Treatment of AEP includes a high-dose of intravenous systemic glucocorticoids until the respiratory

Table 1. Diagnostic criteria for acute eosinophilic pneumonia.

\section{Clinical diagnostic criteria for acute eosinophilic pneumonia}

Febrile illness less than four weeks in duration

Hypoxemic respiratory failure

Diffuse pulmonary opacities on chest radiograph

Bronchoalveolar lavage (BAL) with cell count demonstrating $>25 \%$ eosinophilia

Absence of other known causes for pulmonary eosinophilia 
Table 2. Differential diagnosis considerations for acute eosinophilic pneumonia.

\section{Differential diagnosis considerations}

Eosinophilic granulomatosis with polyangiitis

Pulmonary fungal infections

Pulmonary parasitic infections

Medication effects

Pulmonary infiltration of eosinophils secondary to overwhelming peripheral eosinophilia

failure has resolved, followed by an oral steroid taper. While the optimal duration of a steroid taper has not been determined, generally a slow taper which extends at least two weeks beyond improvements shown by radiographic and laboratory tests is recommended $[1,11]$.

Given the rarity of the disease, most experience with AEP is obtained from retrospective case reports. There is a well-described phenomenon of AEP occurring after new inhalational exposures, including case reports from firefighters [12], military service personnel in the Middle East [13], and first-time cigarette smokers $[3,14]$, and more recently in vaping induced lung disease $[3,4]$.

In the summer of 2019, national attention in the United States [21] was drawn to the dramatic rise in pulmonary toxicity associated with vaping. This is now termed e-cigarette (or vaping) product use-associated lung injury (EVALI) [21].

Case series of EVALI have described a clinical syndrome (Table 3 ) with outcomes ranging from mild selfresolving hypoxemia to refractory hypoxemia requiring extracorporeal membrane oxygenation (ECMO) support $[15,16,17,18]$. There is a broad range of clinical manifestations, including hypersensitivity pneumonitis, diffuse alveolar damage, and organizing pneumonia; however, EVALI manifesting as AEP from THC smoking remains extremely rare. In one of the largest case series of EVALI, a cohort of 98 patients was described, of whom 26 underwent BAL, and none had BAL eosinophilia [15]. In another series of EVALI, all of the forty-three patients underwent BAL. The median eosinophil count was $1 \%$ (range 0-18\%) [15].

A small case series by Maddock et al. (2019) noted large lipid-laden macrophages with red oil staining on BAL cytology samples [16]. This has been postulated to be due to the carrier oils used to dissolve THC. Vitamin $\mathrm{E}$ acetate has been implicated as a potential mechanism for injury due to very high levels found in commercially-available THC vaping products [19]. However, this is difficult to confirm clinically.
Table 3. Characteristics of the clinical syndrome of vapingassociated lung injury.

\section{Differential diagnosis considerations}

Recent e-cigarette use

Pulmonary infiltrates on chest imaging

Absence of pulmonary infection

No evidence of an alternative cause

While our patient's cytology showed small lipidcontaining vacuoles inside macrophages, these are commonly identified as benign cytologic features in any user of inhaled oils and did not match the large macrophages previously described in previous cases. Other potential toxins include THC or nicotine. The majority of EVALI case reports describe the use of THC-containing products (75-80\%), and THC has been found in BAL samples of EVALI patients while it is absent in healthy controls $[15,15,20,21]$. Fewer EVALI patients report exclusive use of nicotine, and 13-58\% of EVALI patients report a history of nicotinevaping $[15,2,21]$. The patient in this current report did not admit to active nicotine use, so there was a higher suspicion for vitamin E oils or THC as the culprit toxin.

While THC has been legalized in some states, it remains illegal in most of the rest of the USA. Thus, there is a substantial market for street-purchased or homemade THC vaping oils, which confounds the ability of regulatory agencies, such as the CDC and FDA, to study and make recommendations about vaping products.

Beyond the recent attention to acute pulmonary toxicity due to vaping, there are concerns regarding the long term safety of vaping. Common vaping flavourings, including glycerin and benzaldehyde, have caused pulmonary toxicity in food factory workers [22] and may be present in e-cigarettes at levels that exceed OSHA limits. Humectants, including glycerin or propylene glycol, result in increased inflammation in vitro human epithelial cells [23]. Furthermore, both flavourings and humectants can undergo pyrolysis or decomposition at high temperatures. Jensen et al. (2015) re- 
ported that commercially available e-liquids undergo pyrolysis in typical use patterns, creating vapour-phase formaldehyde-releasing agents (FRAs) with potential formaldehyde exposure 5-15x higher than traditional tobacco cigarettes [24]. While the International Agency of Research Center classifies formaldehyde as a possible Group 1 human carcinogen, FRAs are not well understood. If FRAs have the same toxicity as gaseous formaldehyde, then long-term health risks of vaping should be monitored with extreme caution.

\section{CONCLUSION}

In summary, a case is presented of acute eosinophilic pneumonia associated with daily use of THC-containing e-cigarettes. Increased vigilance about pulmonary toxicity associated with vaping, mainly vaping THCcontaining products, is essential for clinicians assessing and caring for patients who use e-cigarettes presenting with shortness of breath and respiratory failure.

\section{CONFLICT OF INTEREST}

None to declare.

\section{DEFERENCES}

1. De Giacomi F, Vassallo R, Yi E, Ryu J. Acute Eosinophilic Pneumonia. Causes, Diagnosis, and Management. Am J Respir Crit Care Med. 2018;197:728-36.

2. De Giacomi F, Decker P, Vassallo R, Ryu J. Acute Eosinophilic Pneumonia: Correlation of Clinical Characteristics With Underlying Cause. Chest. 2018;152:379-85.

3. Arter Z, Wiggins A, Hudspath C, Kisling A, Hostler DC, Hostler JM. Acute eosinophilic pneumonia following electronic cigarette use. Respir Med Case Rep. 2019;27:100825.

4. Thota D, Latham E. Case report of electronic cigarettes possibly associated with eosinophilic pneumonitis in a previously healthy active-duty sailor. J Emerg Med. 2014;47(1):15-7.

5. Janz D, O’Neal H, Ely E. Acute eosinophilic pneumonia: A case report and review of the literature. Crit Care Med. 2009;37:1470-74.

6. Cottin V. Eosinophilic Lung Diseases. Clin Chest Med. 2016;37(3):535-56.

7. García-Moguel I, Bobolea I, Diéguez Pastor M, López-Arranz Monge G, Barranco Jimenez R. Acute eosinophilic pneumonia due to piperacillin/tazobactam. Ann Allergy Asthma Immunol. 2019;122:334-36.

8. Kumar S, Acosta-Sanchez I, Rajagopalan N. Daptomycin-induced
Acute Eosinophilic Pneumonia. Cureus. 2018;10(6):e2899.

9. Kwon E, Kim M, Choi E, Park Y, Kim C. Tamoxifen-induced acute eosinophilic pneumonia in a breast cancer patient. Int J Surg Case Rep. 2019;60:186-90.

10. Ohji H, Shinohara T, Kadota N, et al. Pneumocystis jirovecii pneumonia in an HIV-infected patient mimicking acute eosinophilic pneumonia: a case report with a review of the literature. J Thorac Dis. 2018;10:E774-78.

11. Rhee C, Min K, Yim N, Lee J, et al. Clinical characteristics and corticosteroid treatment of acute eosinophilic pneumonia. Eur Respir J. 2013;41(2):402-9.

12. Rom W, Weiden M, Garcia R, et al. Acute eosinophilic pneumonia in a New York City firefighter exposed to World Trade Center dust. Am J Respir Crit Care Med. 2002;166(6):797-800.

13. Shorr A, Scoville S, Cersovsky S, et al. Acute eosinophilic pneumonia among US Military personnel deployed in or near Iraq. JAMA, 2004;292(2):2997-3005.

14. Liu X, Sun W, Meng W, Xiao Y, Feng G, Shi B. Cigarette smokinginduced acute eosinophilic pneumonia: A case report. Med Baltim. 2019;98(9):e14704.

15. Layden J, Ghinai I, Pray I, et al. Pulmonary Illness Related to E-Cigarette Use in Illinois and Wisconsin - Final Report. N Engl J Med. 2020;382:903-16.

16. Maddock S, Cirulis M, Callahan S, et al. Pulmonary Lipid-Laden Macrophages and Vaping. N Engl J Med. 2019;38:1488-89.

17. Henry T, Kanne J, Kligerman S Imaging of Vaping-Associated Lung Disease. N Engl J Med. 2019;381:1486-87.

18. Triantafyllou G, Tiberio P, Zou R, et al. Vaping-Associated Acute Lung Injury: A Case Series. Am J Respir Crit Care Med. 2019;200:1430-31.

19. Centers for Disease Control and Prevention. Outbreak of Lung Injury Associated with E-cigarette. In: Cent. Dis. Control Prev. https://www.cdc.gov/tobacco/basic_information/e-cigarettes/ severe-lung-disease.html. Accessed 9 May 2020.

20. Schier J, Meiman J, Layden J, et al. Severe Pulmonary Disease Associated with Electronic-Cigarette-Product Use - Interim Guidance. MMWR Morb Mortal Wkly Rep. 2019;68:787-90.

21. Centers for Disease Control and Prevention. Outbreak of Lung Injury Associated with the Use of E-Cigarette, or Vaping, Products. https://www.cdc.gov/tobacco/basic_information/ecigarettes/severe-lung-disease.html. Accessed 2 Jul 2020

22. Cummings K, Boylstein R, Stanton M, et al. Respiratory symptoms and lung function abnormalities related to work at a flavouring manufacturing facility. Occup Env Med. 2014;71(8):549-54.

23. Leigh $N$, Lawton R, Hershberger P, Goniewcz M. Flavourings significantly affect inhalation toxicity of aerosol generated from electronic nicotine delivery systems (ENDS). Tob Control. 2016;25:ii81-87.

24. Jensen RP, Luo W, Pankow JF, Strongin RM, Peyton DH. Hidden Formaldehyde in E-Cigarette Aerosols. N Eng J Med. 2015;372:392-394. 